\title{
A mathematical model for evaluating the impact of vaccination schedules: application to Neisseria meningitidis
}

\author{
H. C. TUCKWELL ${ }^{1 *}$, T. HANSLIK ${ }^{1,2}$, A.-J. VALLERON AND A. FLAHAULT ${ }^{1}$ \\ ${ }^{1}$ Epidémiologie et Sciences de l'Information, Inserm U444, Université Pierre et Marie Curie, 27 rue Chaligny, \\ 75571 Paris Cedex 12, France \\ ${ }^{2}$ Service de Médecine Interne, Hôpital Ambroise Paré, Assistance Publique-Hôpitaux de Paris, Université de \\ Versailles, Saint-Quentin-en Yvelines, 9 av Ch de Gaulle, 92100 Boulogne, France
}

(Accepted 28 January 2003)

\section{SUMMARY}

A mathematical model is described which determines the impact of a schedule of vaccination on the time course of a certain class of diseases. The data are the demographic variables and parameters and age-dependent non-fatal and fatal case rates. Given the age- and time-dependent rates of vaccination including coverage and corresponding efficacies, various schedules may be distinguished by either the absolute numbers of cases and deaths avoided or the numbers of cases and deaths avoided per dose of vaccine. The model was applied to meningo-coccal serogroup $\mathrm{C}$ disease in France. The outcomes of six different vaccination schedules were examined. In absolute terms, a schedule in which all individuals aged between 2 and 20 years were vaccinated performed best, but this schedule and that in which only 1 -year olds were vaccinated performed equally and best in terms of cases prevented, but not lives saved, per dose.

\section{INTRODUCTION}

The determination of the effects of schedules of vaccination is an important aspect of epidemiology and public health management. With the emerging threat of bioterrorism, it is also necessary for governments to know the predicted outcomes of emergency vaccination programmes [1].

There are two basic types of model that may be employed in demographic and epidemiological modelling. In the more common type, typified by early SIR models such as those of Kermack-McKendrick [2], the time variable is continuous and the model is formulated as a system of differential equations. Examples of such systems of up to ten components, with application to vaccination against carrier-borne diseases such as tuberculosis are summarized in Bailey [3]. Although continuous time models are often used

\footnotetext{
* Author for correspondence.
}

to make predictions in relation to epidemics and the spread of disease $[4,5]$, they are difficult to employ when there are several age groups.

Alternatively, the time variable is taken to be discrete and the numbers in different age groups are followed throughout the courses of their lives. This approach is typified by the population growth theory developed by Leslie [6] (see [7] for a succinct summary). The numbers of females of reproductive age were updated from epoch to epoch using mortality and fecundity data and several features of the longterm dynamic evolution of the population were deduced from the so-called Leslie matrix. The advantage of this kind of model is that parameters such as birth and death rates correspond to observable quantities and the variables correspond in a precise way with measurable demographic quantities. However, qualitative analysis of equilibria, bifurcations or asymptotic behaviour is more difficult than with differential equation models. 


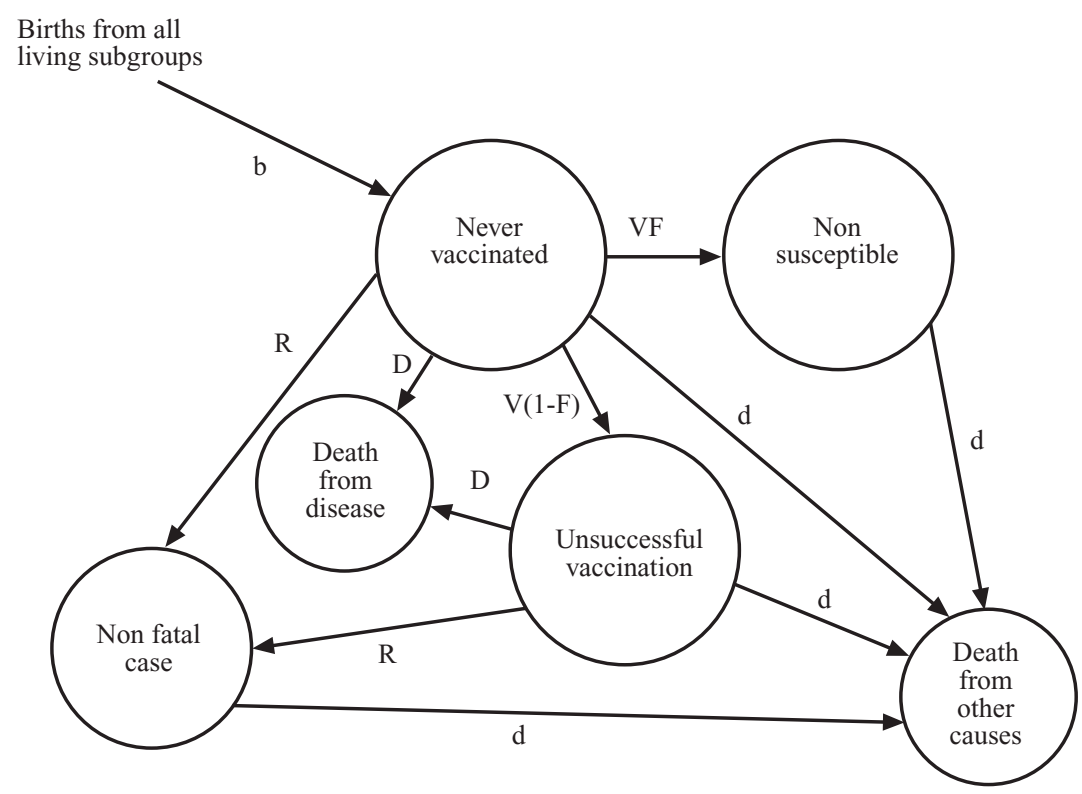

Fig. 1. Relationships between the various subgroups of the population. Symbols attached to arrows are per capita rates per time step. Birth rates are assumed uniform across all living subgroups.

Because we were concerned with measurable quantities, our approach was to formulate a system of difference equations in order to make as accurate as possible yet practically realizable estimates of the effects of various vaccination programmes under certain assumptions about the dynamic structure of the diseased and non-diseased sections of the population. Furthermore, as there has been much recent interest in the prospects of vaccination against meningococcal disease $[8,9]$ and several articles have recently appeared on the cost-effectiveness of various vaccination programmes [10-14], our method is illustrated in Sections 3 and 4 by estimating future case and death rates for meningococcal disease, given various vaccination schedules. The latter embraces both age- and time-dependent components. These are used in conjunction with coverage rates to define a vaccination matrix $V$ which plays a central role in the projections.

\section{THE MODEL}

The population is divided into monthly age groups. Although this subdivision could be finer, the choice of a month seemed appropriate for the chosen application (meningococcal disease). Since the application was designed for humans, those from age 0 to 100 years were included resulting in 1200 age groups, indexed by $j$. The time-steps were also taken to be months, indexed by $k$, with the starting value of $k=1$.

\section{Population with no specific disease and without vaccination}

We let $N_{j k}$ be the number of individuals in the $j$-th age group who are alive at epoch $k$. the overall population birthrate is $b_{k}$ at epoch $k$ with no account for the age of the parents as this is not relevant in the present calculations. In order to extrapolate census data if needed, the age-dependent population numbers are projected as follows. The youngest age group is

$N_{1, k+1}=b_{k} \sum_{j=1}^{1200} N_{j k}$

and for $j>1$,

$N_{j, k+1}=N_{j-1, k}\left(1-d_{j-1, k}\right)+I_{j-1, k}-E_{j-1, k}$

where $I_{j k}$ and $E_{j k}$ are the total immigration and emigration rates and where the $d_{j k}$ are age and timedependent per capita death rates.

\section{Inclusion of a specific disease and vaccination}

For the vaccination of the population, the various population subgroups are as schematized in Figure 1. All living individuals are assumed to belong initially to the never-vaccinated group. Then they may be successfully vaccinated and enter the nonsusceptible group, or unsuccessfully vaccinated and still prone to the disease with either fatal or nonfatal consequences. Any individual at any time may die from causes other than the disease under consideration and the whole 
living population at the end of an epoch may contribute to the population birth processes.

It is assumed that immunity can only be achieved as the result of successful vaccination or becoming a non-fatal case. If natural immunity or immunity induced by other means, (Troncoso et al. [15] report that that Neisseria lactamica may confer immunity to Neisseria meningitidis) is to be included, it may be done by splitting the never-vaccinated group into immune and non-immune. However, vaccination programmes would not distinguish these subgroups.

\section{Definitions of symbols}

The following variables are defined thus:

$A_{j k}$ is the number of individuals in the $j$-th age group at epoch $k$ who have never been vaccinated

$B_{j k}$ is the number of unsuccessfully vaccinated individuals

$C_{j k}$ is the number of nonsusceptibles.

The essential vaccination parameters are contained in the following matrices:

$V_{j k}$, the rate of vaccination of individuals in the $j$-th age group at epoch $k$

$F_{j k}$, the corresponding efficacy rate for the vaccine.

To complete the calculations we specify for the given disease,

$R_{j k}$ the age and time dependent nonfatal case rates $D_{j k}$ the corresponding fatal case rate.

\section{Recursion formulas}

The number of never-vaccinated in the youngest age group consists of all births and is given at epoch $k+1$ in terms of epoch $k$ quantities by

$$
\begin{gathered}
A_{1, k+1}=b(k) \sum_{j=1}^{1200}\left[T_{j k} A_{j k}+\left(1-d_{j k}-D_{j k}\right) B_{j k}\right. \\
\left.+\left(1-d_{j k}\right) C_{j k}\right]
\end{gathered}
$$

where

$T_{j k}=V_{j k}\left[F_{j k}\left(1-d_{j k}\right)+\left(1-F_{j k}\right)\left(1-d_{j k}-D_{j k}\right)\right]$.

The terms here are mainly self explanatory; for example, $\left(1-d_{j k}-D_{j k}\right) B_{j k}$ represents unsuccessfully vaccinated individuals who did not die from other causes or from meningococcal disease; whereas $\left(1-d_{j k}\right) C_{j k}$ represents nonsusceptibles who could not die from the given disease but only from other causes. In (2B), $V_{j k} F_{j k}\left(1-d_{j k}\right)$ comprises successfully vaccinated individuals who do not die from other causes, and $V_{j k}\left(1-F_{j k}\right)\left(1-d_{j k}-D_{j k}\right)$ describes unsuccessfully vaccinated individuals who do not die from either the given disease or from other causes. The numbers of never-vaccinated in the remaining age-groups, $j=2, \ldots, 1200$ are updated according to

$$
\begin{aligned}
A_{j, k+1}= & A_{j-1, k}\left(1-V_{j-1, k}\right) \\
& \times\left(1-d_{j-1, k}-D_{j-1, k}-R_{j-1, k}\right) .
\end{aligned}
$$

Here $\left(1-V_{j-1, k}\right)$ is the unvaccinated fraction of the previously unvaccinated who must survive or not become nonfatal cases.

For the unsuccessfully vaccinated we have for $j=2, \ldots, 1200$,

$$
\begin{aligned}
B_{j, k+1}= & {\left[B_{j-1, k}+\left(1-F_{j-1, k}\right) V_{j-1, k} A_{j-1, k}\right] } \\
& \times\left[1-d_{j-1, k}-D_{j-1, k}-R_{j-1, k}\right] .
\end{aligned}
$$

The nonsusceptibles of the youngest age group are always assumed to be

$C_{1, k+1}=0$,

whereas for $j=2, \ldots, 1200$ we must have

$$
\begin{aligned}
C_{j, k+1}= & C_{j-1, k}\left(1-d_{j-1, k}\right)+A_{j-1, k} \\
& \times\left[F_{j-1, k} V_{j-1, k}\left(1-d_{j-1, k}\right)+\left(1-V_{j-1, k}\right)\right. \\
& \left.\times R_{j-1, k}+V_{j-1, k}\left(1-F_{j-1, k}\right) R_{j-1, k}\right] .
\end{aligned}
$$

To complete the calculations we determine the numbers vaccinated for each age group at each epoch

$N V_{j k}=A_{j k} V_{j k}$

the corresponding number of deaths due to the disease

$N D_{j k}=\left(A_{j k}+B_{j k}\right) D_{j k}$

and the corresponding number of nonfatal cases

$N R_{j k}=\left(A_{j k}+B_{j k}\right) R_{j k}$.

The quantities in (5A)-(5C) are summed over $j$ to obtain total numbers in any epoch. The above system of equations is easily programmable with a software package such as MATLAB.

\section{Allowance for carriers}

In dynamic models of the evolution of a disease in a population it is often important to distinguish between carriers and non carriers [4]. However, in the present quasi-steady state model, the distinction is not necessary if one assumes that the efficacy of the vaccine is the same for carriers and non-carriers, even if the case rates, $\lambda_{1}$ and $\lambda_{2}$, are different for the two groups. Assuming a total population of $N$, a fraction $\rho$ of carriers, and a vaccination rate $v$, the total 
number of cases is just $N\left[\lambda_{1} \rho+(1-\rho) \lambda_{2}\right]$ without vaccination and $(1-v)$ times this quantity following vaccination. This follows because vaccination is assumed to immunize both susceptible (non-diseased) carriers and susceptible non-carriers.

\section{APPLICATION TO MENINGOCOCCAL DISEASE}

There are 12 distinct serogroups of Neisseria meningitidis, those causing most disease being $\mathrm{A}, \mathrm{B}, \mathrm{C}$, $\mathrm{W}-135$ and $\mathrm{Y}$ [16]. In most industrialized countries the most common serogroup responsible for disease is $\mathrm{B}$, followed by $\mathrm{C}$.

At the time of writing, there exist capsular polysaccharide vaccines for the serogroups A, C, W-135 and $\mathrm{Y}$ but these are not indicated for children below 2 years of age, because they are poorly effective in this age group. Furthermore, these vaccines only provide a duration of protection of no more than 5 years in older children and adults. There is no vaccine for serogroup B, but one may be soon forthcoming with the use of DNA sequencing [17].

\section{The role of carriers}

The bacteria responsible for meningococcal disease are found in a considerable percentage of the population who are carriers but have no illness. Evidently, the bacteria may reside in the nasopharynx without penetrating the body systems [18]. The average carriage rate summed over all identifiable and nontypable serogroups is probably of order $5-10 \%$ [19] with one report claiming that rates were $10 \%$ in adults and $30 \%$ in teenagers [16].

The rates of carriage of Neisseria meningitidis are highly variable and may show dramatic changes when social conditions change. Thus for example, in a group of first-year British college students, the rate of carriage grew from $7 \%$ in the population of entering students to $23 \%$ in just 4 days and continued to grow to reach a maximum of $30 \%$ after several months [20]. The dynamics of transitions between the carrier state and the non-carrier, undiseased state are however not known. For schoolchildren in Belgium the mean duration of carriage of $N$. meningitidis was estimated to be about 13 months [18].

Rates of carriage for serogroup $\mathrm{C}$ are usually relatively low, outbreaks of disease being attributed to the rapid spread of virulent strains through susceptible subgroups of the population such as teenagers starting college or university, recruits entering military training camps and infants beginning school or daycare [16]. During such periods there are increased acquisition rates. In a recent study related to the comprehensive vaccination in the UK [21], the rate of carriage of serogroup $\mathrm{C}$ in school students aged between 15 and 17 years was estimated at $0.45 \%$ in 1999 and $0.15 \%$ in the post-vaccination year 2000. In 2000 the carriage rate of $0 \cdot 127 \%$ in vaccinated students was significantly less than the rate of $0.342 \%$ in unvaccinated students, the latter figure supporting the idea that there may be some degree of "herd immunity'.

Since the details of transitions from carrier to diseased or from non-carrier to diseased state were unknown for $N$. meningitidis it was decided to assume the rates of incidence of fatal and nonfatal cases were given by the available age-dependent rates.

The explicit division of the uninfected population into carriers and noncarriers was ignored. The model assumed a steady state with carriers and noncarriers in equilibrium, and in which the per capita rates of fatal and nonfatal cases was fixed, thus presupposing that these rates were small relative to the overall death and birth rates, so that carriage rates do not change significantly. The latter assumption is valid for meningococcal disease in Western countries where the overall death and birth rates are of the order of 0.01 per year, whereas the disease rate is usually of order of 0.00001 per year. With regard to the impact of vaccination, the model implicitly yet heuristically takes account of the distinction between carriers and noncarriers.

\section{Application of the model to serogroup C meningococcal disease}

The recently developed capsular polysaccharidecarrier protein conjugate vaccines for invasive disease due to meningococcal serogroup $\mathrm{C}$ may be successfully administered to children as young as 2 months of age. Faced with an endemic of invasive meningococcal serogroup $\mathrm{C}$ disease, with an elevated incidence in children below 5 years of age and a high casefatality rate among adolescents ( 15 to 20 years of age), the United Kingdom launched a mass immunization programme for children from 2 months to 17 years of age with capsular polysaccharide-carrier protein vaccines in November 1999. There followed a dramatic decrease in the incidence of serogroup $\mathrm{C}$ disease $[21,22]$. 


\section{Demographic and epidemiological data}

The census data for France (www.recensement. insee.fr) at the end of 1999 were first extrapolated for 2 years to give the beginning of 2002 as the start time. The population numbers in yearly age groups and corresponding death rates from 0 to 100 years were converted to monthly figures. The population birth rate was set at the most recently available figure of 0.0127 per year. The yearly age distribution of death rates for meningococcal $\mathrm{C}$ disease for the years 1985-2000 was obtained from the web site for the Institut de Veille Santé weekly publications (www. invs.sante.fr/beh/2002/25/BEH25.htm) and also converted to monthly figures. No account was taken of seasonal variations.

\section{Vaccination schedules}

We examined several schedules of vaccination, some involving narrow age groups and others spanning many years. The age of the recipient also affects frequency of dosage: for this particular vaccine, infants under 6 months receive three doses in successive months, infants between 6 months and 1 year receive two doses, whereas individuals older than 12 months are given only one dose. This has a large effect on the cost of vaccinating in the very young age groups. With certain vaccines there is a possibility that individuals vaccinated as infants may need a booster at a later age (for the case of Hib see [23]) - this may affect the schedules A and B described below but was not included in the calculations.

The programmes examined were as follows, where we included the two extreme cases $\mathrm{O}$, in which nobody was vaccinated, and $\mathrm{R}$, in which the entire population, from age 1 month to 100 years, was successfully vaccinated with the number of doses appropriate for different ages:

O No vaccination

A Three doses at 2, 3 and 4 months

B One dose at 12 months

$\mathrm{C}$ One dose at 1 year, 6 years and 11 years for 5 years, followed by schedule $A$

$\mathrm{D}$ One dose at 1 year, 6 years and 11 years for 5 years, followed by schedule $B$

$\mathrm{E}$ All individuals from 1 year to 20 years

$\mathrm{F}$ All individuals from 2 months of age to 20 years

$\mathrm{R}$ Vaccination of the entire population

Note that, for example, in schedule B, the dose at 1 year is taken literally; that is, vaccination takes place on entering the 13 th month of life. It is not assumed to mean all 1-year olds which might usually be interpreted as all infants with ages greater than 12 months and less than 24 months. However, the programme can easily accommodate different interpretations of all one year olds by changing the elements of the vaccination matrix.

\section{Efficacy}

The vaccination of an individual may not lead to immunity so that the efficacy, or fraction successfully protected against disease is less than 1. In all of the calculations we have done for Neisseria meningitidis serogroup $\mathrm{C}$, the choices of values for efficacy were guided by reference [24]. Thus for all $k$, for toddlers,

$F_{j k}=0.92, \quad j=1, \ldots, 24 ;$

for between toddler and teenager,

$F_{j k}=0.92+0.05(j-24) / 156, \quad j=25, \ldots, 179$

and for teenager and older

$F_{j k}=0.97, \quad j>179$.

\section{Vaccination matrix and coverage}

The vaccination matrix $V$ characterizes the schedule of vaccination. For example, for schedule B, which sees every 1 -year-old vaccinated on achieving that age, we put

$V_{13, k}=1, \quad k \geqslant 1$

with all other $V_{j k}=0$. However, this assumes that every individual in a target group is vaccinated. In practice, this is not the case. For vaccination against meningococcal disease due to serogroup $\mathrm{C}$ the actual rate of vaccination in the UK programme depended on the age group [24]. We were guided by the data of Miller et al. [22] and set the coverage at $85 \%$ regardless of target group. Thus instead of $V_{13, k}=1, k \geqslant 1$, for schedule B we put $V_{13, k}=0 \cdot 85, k \geqslant 1$, for the nonzero entries. Note that the programme automatically excludes the possibility of multiple vaccinations, (apart from cases where multiple doses are specified) for example at 12 months and at 5 years, as once

Table 1. Schedule O: no individuals vaccinated

\begin{tabular}{lrrr}
\hline \hline & 1 year & 5 years & 10 years \\
\hline Total number of cases & 219 & 1098 & 2203 \\
Total number of deaths & 33 & 165 & 330 \\
\hline \hline
\end{tabular}


Table 2. Schedule A: 3 doses at 2, 3 and 4 months

\begin{tabular}{lccc}
\hline \hline & 1 year & 5 years & 10 years \\
\hline Total number of cases & 213 & 955 & 1714 \\
Number of cases avoided & 6 & 145 & 489 \\
Total number of deaths & 32 & 152 & 284 \\
Number of deaths avoided & 1 & 13 & 46 \\
Total number of doses (millions) & $1 \cdot 92$ & $9 \cdot 68$ & $19 \cdot 5$ \\
Cases avoided per million doses & $3 \cdot 13$ & 14.98 & $25 \cdot 08$ \\
Deaths avoided per million doses & 0.52 & 1.34 & $2 \cdot 36$ \\
\hline \hline
\end{tabular}

Table 3. Schedule B: 1 dose at 12 months

\begin{tabular}{lccc}
\hline \hline & 1 year & 5 years & 10 years \\
\hline Total number of cases & 214 & 968 & 1768 \\
Number of cases avoided & 5 & 132 & 435 \\
Total number of deaths & 33 & 154 & 290 \\
Number of deaths avoided & 0 & 11 & 40 \\
Total number of doses (millions) & $0 \cdot 64$ & $3 \cdot 21$ & $6 \cdot 46$ \\
Cases avoided per million doses & $7 \cdot 81$ & $41 \cdot 12$ & $67 \cdot 34$ \\
Deaths avoided per million doses & 0 & $3 \cdot 43$ & $6 \cdot 19$ \\
\hline \hline
\end{tabular}

Table 4. Schedule C: 1 dose at 1, 6 and 11 years for 5 years; then schedule $A$

\begin{tabular}{lccc}
\hline \hline & 1 year & 5 years & 10 years \\
\hline Total number of cases & 209 & 858 & 1411 \\
Number of cases avoided & 10 & 242 & 792 \\
Total number of deaths & 32 & 138 & 234 \\
Number of deaths avoided & 1 & 27 & 96 \\
Total number of doses (millions) & $1 \cdot 88$ & $8 \cdot 21$ & $18 \cdot 93$ \\
Cases avoided per million doses & $5 \cdot 32$ & $26 \cdot 28$ & $41 \cdot 84$ \\
Deaths avoided per million doses & $0 \cdot 53$ & $2 \cdot 93$ & $5 \cdot 07$ \\
\hline \hline
\end{tabular}

Table 5. Schedule D: 1 dose at 1, 6 and 11 years for 5 years; then schedule B

\begin{tabular}{lccc}
\hline \hline & 1 year & 5 years & 10 years \\
\hline Total number of cases & 209 & 858 & 1424 \\
Number of cases avoided & 10 & 242 & 779 \\
Total number of deaths & 32 & 138 & 236 \\
Number of deaths avoided & 1 & 27 & 94 \\
Total number of doses (millions) & $1 \cdot 88$ & $8 \cdot 21$ & $12 \cdot 48$ \\
Cases avoided per million doses & $5 \cdot 32$ & $26 \cdot 28$ & $62 \cdot 42$ \\
Deaths avoided per million doses & $0 \cdot 53$ & $2 \cdot 93$ & $7 \cdot 53$ \\
\hline \hline
\end{tabular}

vaccinated, an individual is taken out of all target groups in the subsequent epoch.

The numerical results for the various schedules are shown in Tables 1-8. It was assumed that the vaccination is in fact effective for up to 10 years, although a lesser period of effectiveness could be incorporated by insisting that living previously vaccinated individuals re-enter the susceptible subgroup after a certain number of epochs, possibly in a graded or stochastic fashion.

The results of the tables are also presented graphically as follows. In Figure 2 we show the numbers of deaths due to meningococcal disease serogroup $\mathrm{C}$ which are avoided when the various schedules $\mathrm{A}-\mathrm{F}$ and $\mathrm{R}$ are applied without cessation for 10 years. In Figure 3 are shown the corresponding numbers 
Table 6. Schedule E: all individuals from 1 year to 20 years

\begin{tabular}{lccc}
\hline \hline & 1 year & 5 years & 10 years \\
\hline Total number of cases & 82 & 362 & 737 \\
Number of cases avoided & 137 & 738 & 1466 \\
Total number of deaths & 14 & 62 & 123 \\
Number of deaths avoided & 19 & 103 & 207 \\
Total number of doses (millions) & $15 \cdot 52$ & $17 \cdot 95$ & $21 \cdot 21$ \\
Cases avoided per million doses & $8 \cdot 83$ & $41 \cdot 11$ & $69 \cdot 12$ \\
Deaths avoided per million doses & $1 \cdot 22$ & $5 \cdot 74$ & $9 \cdot 76$ \\
\hline \hline
\end{tabular}

Table 7. Schedule F: all individuals from 2 months to 20 years

\begin{tabular}{lccc}
\hline \hline & 1 year & 5 years & 10 years \\
\hline Total number of cases & 72 & 300 & 610 \\
Number of cases avoided & 147 & 800 & 1593 \\
Total number of deaths & 13 & 56 & 110 \\
Number of deaths avoided & 20 & 109 & 220 \\
Total number of doses (millions) & $18 \cdot 11$ & $25 \cdot 91$ & $35 \cdot 72$ \\
Cases avoided per million doses & $8 \cdot 11$ & $30 \cdot 88$ & $44 \cdot 60$ \\
Deaths avoided per million doses & $1 \cdot 10$ & $4 \cdot 21$ & $6 \cdot 16$ \\
\hline \hline
\end{tabular}

Table 8. Schedule R: all individuals

\begin{tabular}{lccc}
\hline \hline & 1 year & 5 years & 10 years \\
\hline Total number of cases & 0 & 0 & 0 \\
Number of cases avoided & 219 & 1100 & 2203 \\
Total number of deaths & 0 & 0 & 0 \\
Number of deaths avoided & 33 & 165 & 330 \\
Total number of doses (millions) & 60 & 63 & 67 \\
Cases avoided per million doses & $3 \cdot 65$ & $17 \cdot 46$ & $32 \cdot 88$ \\
Deaths avoided per million doses & $0 \cdot 55$ & $2 \cdot 82$ & 4.93 \\
\hline \hline
\end{tabular}

of cases avoided per million doses of vaccine and Figure 4 shows the numbers of deaths avoided per million doses of vaccine. Figure 2 enables a rapid consideration as to how the various schedules differ in their consequences with regard to absolute numbers of lives saved. A similar figure may be drawn for cases avoided. Figures 3 and 4 are useful for economic considerations as they yield an assessment of the comparative efficiencies, in terms of cases or deaths avoided per million doses of vaccine, for various schedules.

It is also of interest to examine the time course of monthly case and death rates after a given vaccination programme has been instigated. To save space the only results to be displayed graphically here are for schedule D. Figure 5 shows the age distribution of deaths per month due to meningococcal disease serotype $\mathrm{C}$ under this schedule after it has been applied for 1 year, 5 years and 10 years. The death rate for individuals less than 1 year old is always unchanged. After 1 year, there are gaps in the distribution due to the vaccination of the 1, 6 and 11 year olds and after 5 years one can see the widening of the gaps as the vaccinated individuals become older. This continues to 10 years but there is a residual death rate due to the fact that the efficacy is less than $100 \%$.

\section{DISCUSSION AND CONCLUSIONS}

We formulated a discrete time model for the effects of vaccination on a certain class of diseases. The class of 


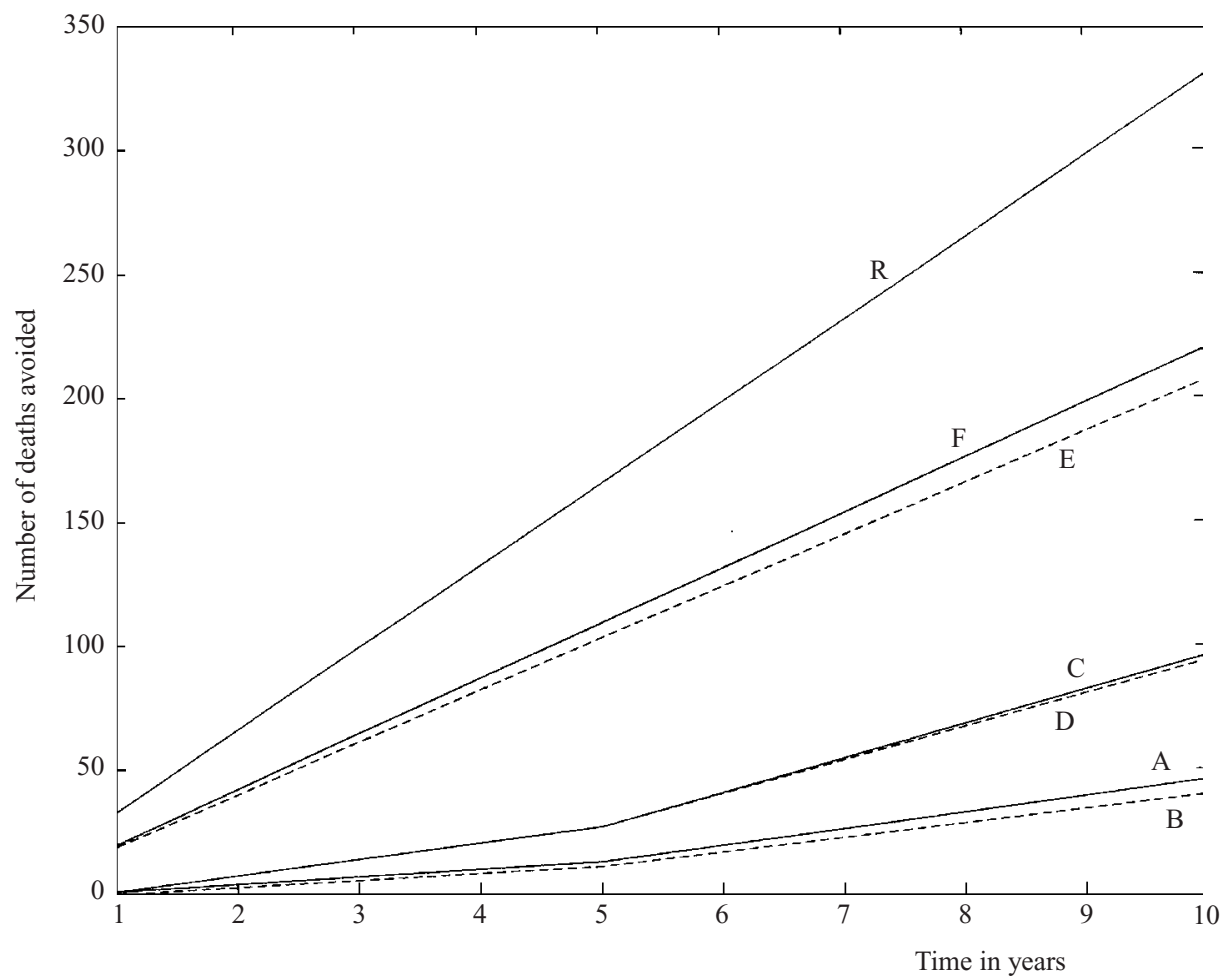

Fig. 2. Results of applying the various vaccination schedules $\mathrm{A}-\mathrm{F}$ and the reference $\mathrm{R}$ consisting of vaccinating the entire population. The number of deaths avoided is plotted against time, assuming that the schedules are applied continuously.

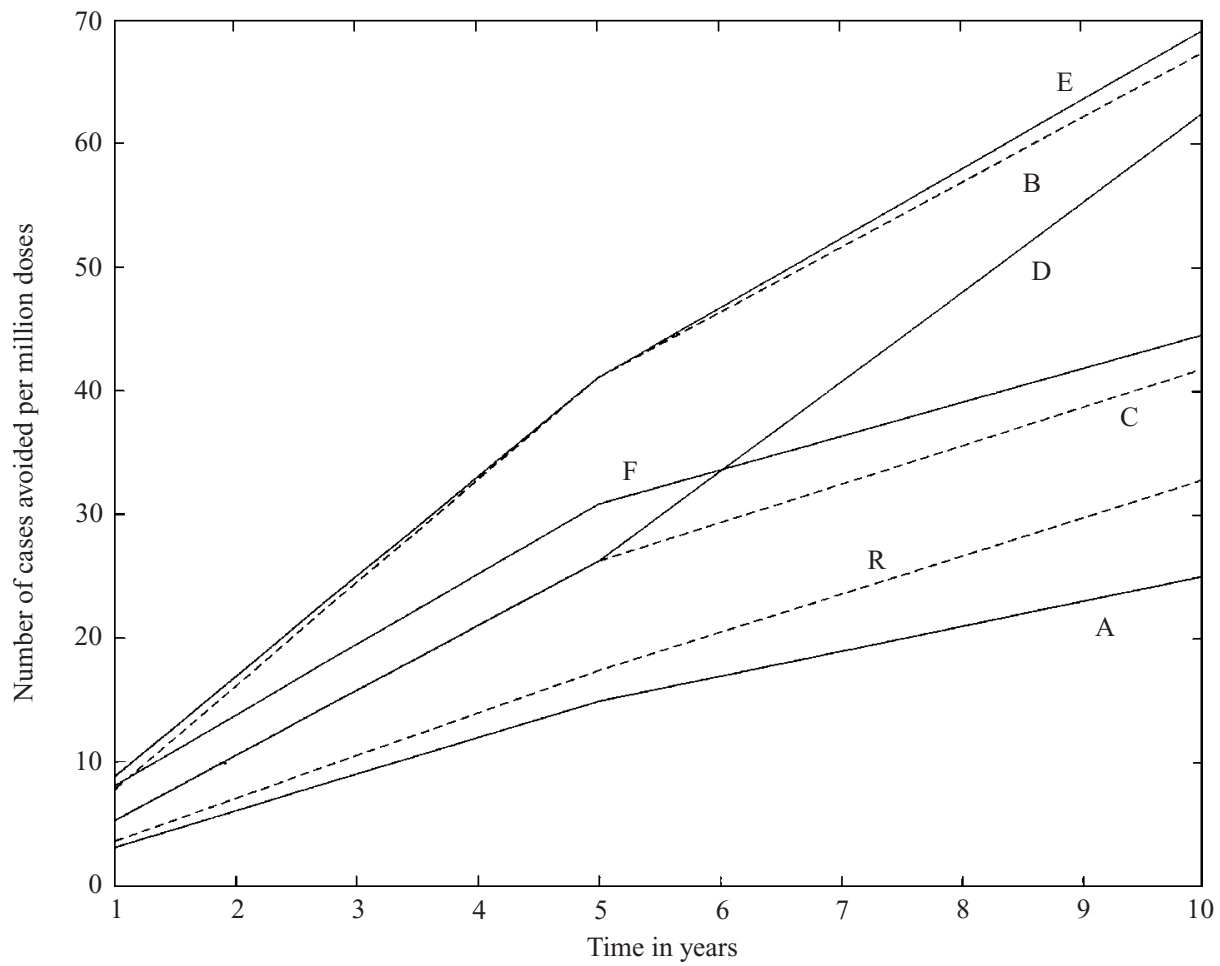

Fig. 3. The number of cases avoided due to serogroup $\mathrm{C}$ meningococcal disease for each one million doses of vaccine plotted against time for each vaccination schedule. 


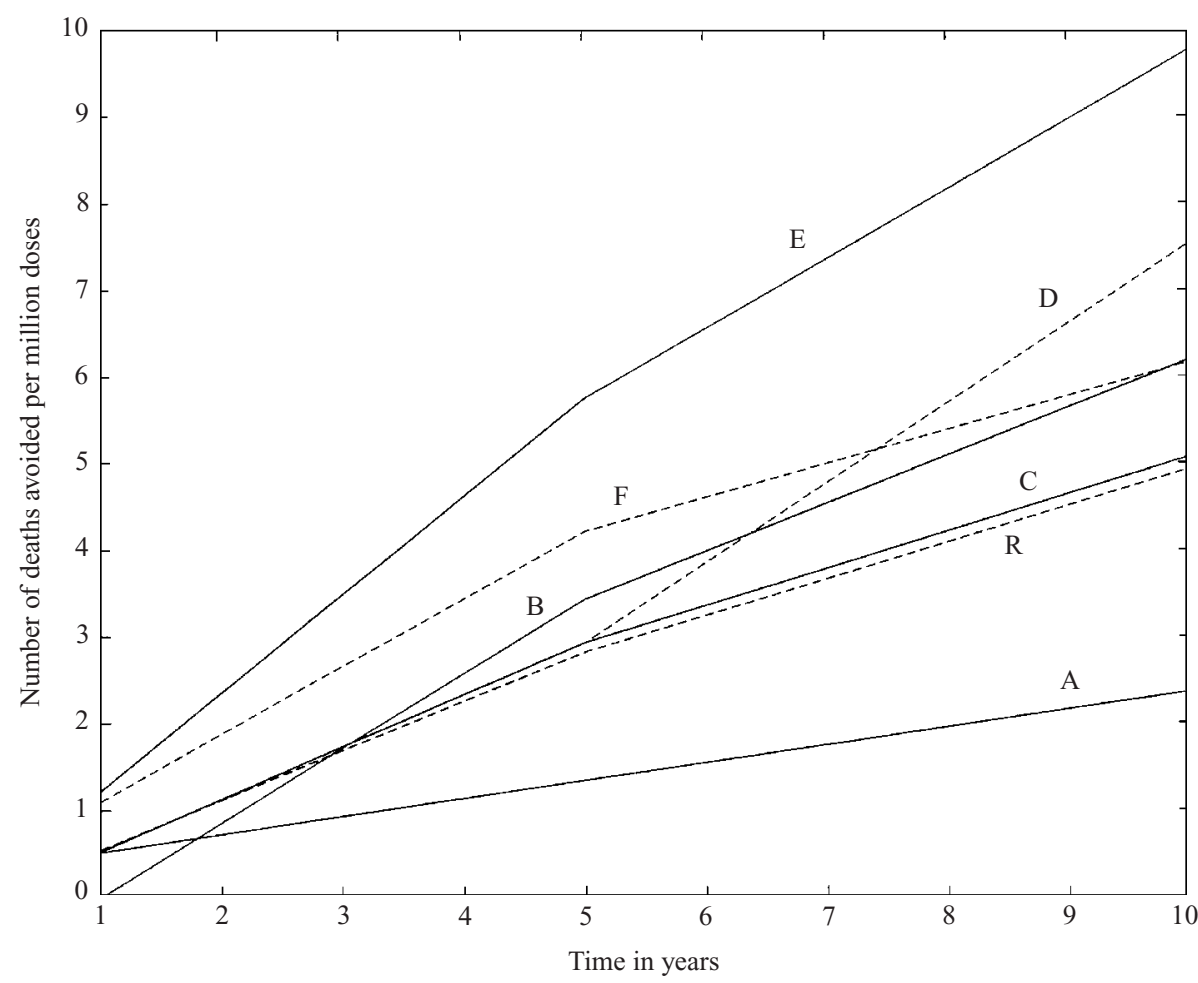

Fig. 4. The number of deaths of serogroup $\mathrm{C}$ meningococcal disease avoided per million doses, plotted against time for each schedule of vaccination.
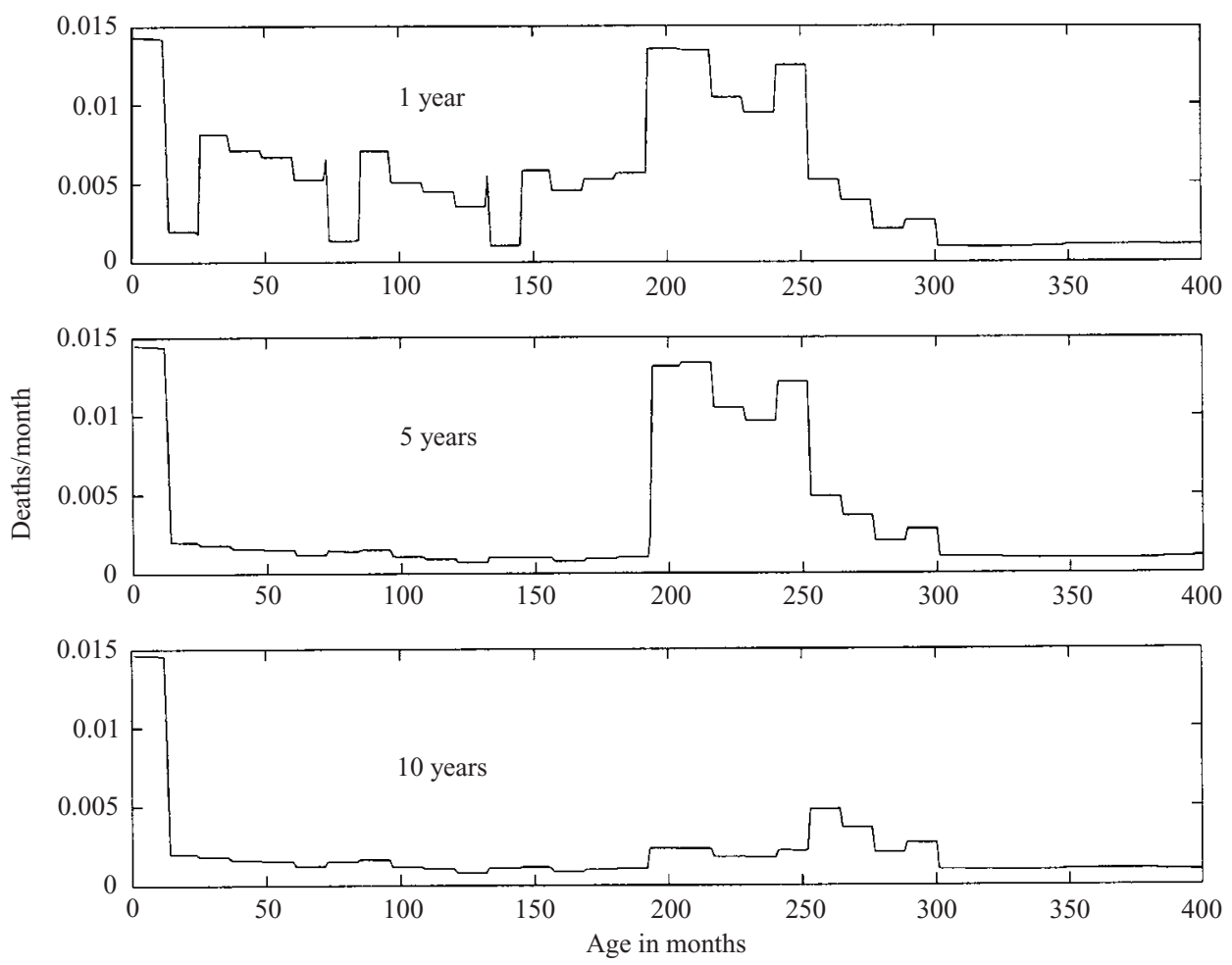

Fig. 5. The numbers of deaths per month as a function of age for progressive stages of vaccination according to schedule D. 
diseases is quite broad if reasonably accurate case and fatality rates are known as functions of time. However, since these rates may be difficult to predict, the most useful application of the model is to diseases for which the rates are not changing too rapidly. The ideal application is to a population in which the disease afflicts a known fraction in each age group in each time period. However, the model could be extended to diseases where contagion leads to changing infection rates if the latter can be estimated in an susceptible-infected homogeneous mixing framework.

The discrete-time projection method offers the advantages of explicit interpretations for parameters and accurate forecasting. In particular in the present model, vaccination rates, vaccination efficacies, disease incidence both fatal and nonfatal, and death rates from other causes are all age-, and possibly timedependent. The model has been implemented without difficulty with standard mathematical software packages. The model was used to explore the possible effects of vaccinating against meningococcal disease serogroup $\mathrm{C}$ in the French population. Six different vaccination schedules were analysed and also the effects of vaccinating the entire population so that a comparison could be made with the 'best case' scenario.

For each schedule we determined, as functions of time, the number of meningococcal type $\mathrm{C}$ deaths avoided, the number of non-fatal cases avoided, the number of doses required, and the number of lives saved and cases avoided per million doses of vaccine, taking into account multiple doses in the youngest age groups. From the latter calculated quantities, if the cost per dose is known as say $\$ X$, then the financial cost involved in saving one life or avoiding one case can be determined easily. For example, for schedule A run for 10 years, the cost to save one life is about $\$ 400000 X$ whereas the corresponding cost for schedule $\mathrm{E}$ is about $\$ 100000 X$. Note that the results presented in Figures 2-5 reflect the epidemiology of invasive meningococcal $\mathrm{C}$ disease vis a vis the elevated incidence in infants and a peak in mortality in adolescent years.

With regard to a schedule that will maximize the numbers of lives saved and cases avoided, it is clear that the more individuals are vaccinated the better to a certain extent, but that it is important if the number of doses is fixed that vaccinating the age-groups with the greatest incidence will lead to the greatest benefit. If vaccine is in limited supply then the greatest benefit does not necessarily derive from pursuing a schedule that prevents the most cases in absolute terms. The reason seems to be that doses can be wasted on groups with small risk.

Thus, in Figure 1 it can be seen that schedule B (vaccinate at one year) performs worst with zero deaths avoided in the first year, 11 after 5 years and 40 after 10 years. Apart from schedule R (whole population vaccinated) the best in absolute terms of deaths and cases avoided is $\mathrm{F}$, in which all individuals from 2 years to 20 years are vaccinated. In absolute terms, schedules $\mathrm{F}$ and $\mathrm{E}$ both prevent about $65 \%$ of the maximum attainable, whereas schedules $\mathrm{C}$ and $\mathrm{D}$ (1 year, 6 years and 11 years for 5 years, followed by $\mathrm{A}$ or B respectively) prevent only about $16 \%$ of the maximum number for deaths after 5 years but about $28 \%$ after 10 years.

However, if the total dosage is limited, we should examine the graphs shown in Figures 3 and 4. In Figure 3, where the number of cases avoided per dose is graphed, we see that schedule E performs best but that it is very closely mimicked by $\mathrm{B}$, which has the worst rating in absolute terms. Furthermore, schedule D approaches after 7 or so years the efficiency of schedules E and B. Similarly, in Figure 4, it is seen that on a per number of doses basis, schedule $\mathrm{E}$ in which all individuals between 2 and 20 are vaccinated, performs best in terms of deaths avoided due to meningococcal disease type $\mathrm{C}$. The second most effective schedule using this criterion is $\mathrm{F}$ for the first 7 years but this is overtaken by D in years $7-10$. Interestingly, schedule $\mathrm{R}$ is only about $50 \%$ as efficient as E.

The difference between the performances in Figures 3 and 4 are due to the fact that the age-distribution of fatal and non-fatal cases are different.

In conclusion, in absolute terms, the best schedule apart from $\mathrm{R}$ is $\mathrm{E}$ and the worst is $\mathrm{B}$; but in terms of value per dose, $\mathrm{B}$ and $\mathrm{E}$ are best and $\mathrm{A}$ is worst. The above calculations, although deterministic both with regard to demography and the processes of disease and vaccination, are thus useful in choosing between vaccination schedules with regard to absolute frequencies of cases and deaths due to a disease, or with regard to particular public health criteria for the implementation of a new vaccination schedule. These calculations do not take into account the public and professional susceptibility of any particular vaccination schedule, nor the practicality of integration of any new vaccine into already-established programmes. 


\section{ACKNOWLEDGEMENTS}

HCT gratefully acknowledges support from INSERM and the hospitality at U444. We appreciate the useful remarks on the manuscript by Dr Mark Fletcher of Wyeth.

\section{REFERENCES}

1. Kaplan EH, Craft DL, Wein LM. Emergency response to a smallpox attack: the case for mass vaccination. Proc Natl Acad Sci USA 2002; 99: 10935-42.

2. Kermack WO, McKendrick AG. Contributions to the mathematical theory of epidemics I. Proc Roy Soc Lond A $1927 ; 11: 700-21$.

3. Bailey NTJ. The mathematical theory of infectious diseases and its application. London: Griffin, 1975.

4. Coen PG, Heath PT, Barbour ML, Garnett GP. Mathematical models of Haemophilus influenzae type b. Epidemiol Infect 1998; 120: 281-95.

5. Hethcote HW. The mathematics of infectious diseases. SIAM Review 2000; 42: 599-653.

6. Leslie PH. On the use of matrices in certain population mathematics. Biometrika 1945; 33: 183-212.

7. Pollard JH. Mathematical models for the growth of human populations. Cambridge: CUP, 1973.

8. Lingappa JR, Rosenstein N, Zell ER, Shutt KA, Schuchat A, Perkins BA. Surveillance for meningococcal disease and strategies for use of conjugate meningococcal vaccines in the United States. Vaccine 2001; 29: 4566-75.

9. Jodar L, Feavers IM, Salisbury D, Granoff DM. Development of vaccines against meningococcal disease. Lancet $2002 ;$ 359: 1499-508.

10. du Chatelet IP, Gessner BD, da Silva A. Comparison of cost-effectiveness of preventive and reactive mass immunization campaigns against meningococcal meningitis in West Africa: a theoretical modelling analysis. Vaccine $2001 ; 19$ : 3420-31.

11. Bos JM, Rumke HC, Welte R, Postma MJ, Jager JC. Health economics of a hexavalent meningococcal outermembrane vesicle vaccine in children: potential impact of introduction in the Dutch vaccination program. Vaccine 2002; 20: 202-07.

12. De Wals P, Erickson L. Economic analysis of the 19921993 mass immunization campaign against serogroup C meningococcal disease in Quebec. Vaccine 2002; 20: 2840-4.

13. Trotter CL, Edmunds WJ. Modelling cost effectiveness of meningococcal serogroup $\mathrm{C}$ conjugate vaccination campaign in England and Wales. BMJ 2002; 324: 809-12.

14. Scott RD, Meltzer MI, Erickson LJ, De Wals P, Rosenstein NE. Vaccinating first-year college students living in dormitories for meningococcal disease. An economic analysis. Am J Prev Med 2002; 23: 98-105.

15. Troncoso G, Sanchez S, Criado MT, Ferreiros CM. Analysis of Neisseria lactamica antigens putatively implicated in acquisition of natural immunity to Neisseria meningitidis. FEMS Immunol Med Microbiol 2002; 1417: 1-7.

16. Morley SL, Pollard AJ. Vaccine prevention of meningococcal disease, coming soon? Vaccine 2001; 20: 666-87.

17. Poolman J, Berthet F-X. Alternative vaccine strategies to prevent serogroup B meningococcal diseases. Vaccine 2002; 20 : S24-6.

18. Coen PG, Cartwright K, Stuart J. Mathematical modelling of infection and disease due to Neisseria meningitidis and Neisseria lactamica. Int $\mathbf{J}$ Epidemiol 2000; 29: 180-8.

19. Benenson AS, ed. Control of communicable diseases manual. Washington, DC: APHA, 1995.

20. Neal KR, Nguyen-Van-Tam JS, Jeffrey N, et al. Changing carriage rate of Neisseria meningitidis among university students during the first week of term: cross sectional study. BMJ 2000; 320 : 846-9.

21. Maiden MCJ, Stuart JM. Carriage of serogroup C meningococci 1 year after meningococcal $\mathrm{C}$ conjugate polysaccharide vaccination. Lancet 2002; 359: 1829-30.

22. Miller E, Salisbury D, Ramsay M. Planning, registration, and implementation of an immunisation campaign against meningococcal serogroup $\mathrm{C}$ disease in the UK: a success story. Vaccine 2002; 20: S58-67.

23. Heath PT, Booy R, Azzopardi HJ, et al. Antibody concentration and clinical protection after Hib conjugate vaccination in the United Kingdom. JAMA 2000; 284: 2334-40.

24. Ramsay ME, Andrews N, Kacsmarski EB, Miller E. Efficacy of meningococcal serogroup C conjugate vaccine in teenagers and toddlers in England. Lancet 2001; 357: 195-6. 